HUTP-98/A068

hep-th/9809199

\title{
Quantum Field Theory of Treasury Bonds
}

\author{
Belal E. Baaquie' \\ Lyman Laboratory of Physics \\ Harvard University \\ Cambridge, MA 02138, U.S.A.
}

\begin{abstract}
The Heath-Jarrow-Morton (HJM) formulation of treasury bonds in terms of forward rates is recast as a problem in path integration. The HJM-model is generalized to the case where all the forward rates are allowed to fluctuate independently. The resulting theory is shown to be a two-dimensional Gaussian quantum field theory. The no arbitrage condition is obtained and a functional integral derivation is given for the price of a futures and an options contract. PACS:02.50.-r Probability theory, stochastic processes $05.40 .+\mathrm{j}$ Fluctuation phenomena, random processes and Brownian motion 03.05.-w Quantum mechanics
\end{abstract}

\footnotetext{
${ }^{1}$ Permanent address: Department of Physics, National University of Singapore, Kent Ridge Road, Singapore 091174; e-mail:phybeb@nus.edu.sg
} 


\section{Introduction}

Stochastic calculus is the most widely used mathematical formalism for modeling financial instruments [1], followed by the use of partial differential equations [2]. The Feynman path integral is a formalism based on functional integration and is widely used in theoretical physics to model quantum (random) phenomenon [3]; it is also ideally suited for studying stochastic processes aris-

ing in finance. In [4 techniques from physics were applied to the study of finance and in [5] the problem of the pricing of stock options with stochastic volatility was studied using the formalism of path integration.

In this paper, the path integral approach is continued into the field of interest rates embodied in the modeling of Treasury bonds. The complexity of this problem is far greater than that encountered in the study of stocks and their derivatives; the reason being that a stock at a given instant in time is described by only one stochastic variable undergoing random evolution whereas in the case of the interest rates it is the entire yield curve which is randomly evolving and requires infinitely many independent variables for its description. The theory of quantum fields [6] has been developed precisely to study problems involving infinitely many variables and so we are naturally led to the techniques of quantum field theory in the study of the interest yield curve.

Treating all the forward rates as independent random variables has also been studied in [7, 8, 9, 10] using the formalism of stochastic calculus. In this approach a stochastic partial differential equation in infinitely many variables is written. The approach based on quantum field theory is in some sense complimentary to the approach based on stochastic partial differential equations since the expressions for all financial instruments are formally given as a functional integral. One advantage of the approach based on quantum field theory is that the introduction of non-linearities as well as stochastic volatility is easily incorporated.

The HJM-model [11] is taken as the starting point of this paper. In Section 2 the HJM-model is re-expressed in terms of a path integral, and the condition of no arbitrage is re-derived in this formalism. To make the formalism more transparent and accessible to readers not familiar with path integration, the well-known results for the price of futures of zero-coupon bonds as well as the price of a European call option and a cap for a zero-coupon bond is derived in Sections 3 and 4 respectively. Another more important reason for these 
re-derivations is that the prices of these derivatives are expressed in a form which can be directly generalized to the case when we model the evolution of the forward rates using quantum field theory.

In Section 5, the HJM-model is generalized to the case with independent fluctuations of all the forward rates; the theory is then seen to consist of a free (Gaussian) two-dimensional quantum field theory. The generalized model has a new parameter which determines how strongly it deviates from the HJM-model. The condition of no arbitrage is derived for the generalized model.

In Section 6, the formulae for the prices of futures and options of zerocoupon bonds are obtained explicitly for the Gaussian quantum field theory.

In Section 7 some conclusions are discussed as well as possible future directions of research.

\section{Path Integral Formulation of the HJM-model}

Bonds are financial instruments of debt which are issued by governments and corporations to raise money from the capital markets [12, 13]. Bonds have a predetermined (deterministic) cash flow; a Treasury bond is an instrument for which there is no risk of default in receiving the payments, whereas for corporate bonds there is in principle such a risk. A Treasury zero-coupon bond is a risk-free financial instrument which has a single cash-flow consisting of a fixed pay-off of say $\$ 1$ at some future time $\mathrm{T}$; its price at time $t<T$ is denoted by $P(t, T)$, with $P(T, T)=1$.

A Treasury coupon bond $\mathcal{B}(t, T)$ has a series of predetermined cash-flows which consists of coupons worth $c_{i}$ paid out at increasing times $T_{i}$ 's, and with the principal worth $L$ being paid at time $T \cdot \mathcal{B}(t, T)$ is given in terms of the zero-coupon bonds by 12

$$
\mathcal{B}(t, T)=\sum_{i=1}^{K} c_{i} P\left(t, T_{i}\right)+L P(t, T)
$$

From above we see that a coupon bond is equivalent to a portfolio of zero-coupon bonds. Hence, if we model the behaviour of zero-coupon bonds, we automatically have a model for coupon bonds as well

Consider the forward rate $f(t, x)$, which stands for the spot (overnight) interest rate at future time $x$ for a contract entered into at time $t<x$. The 
price of a zero-coupon bond with the value of $\$ 1$ at maturity is given by

$$
P(t, T)=\exp \left\{-\int_{t}^{T} d x f(t, x)\right\}
$$

Note from its definition, the spot rate for an overnight loan at some (future) time $t$ is $r(t)$ and is given by

$$
r(t)=f(t, t)
$$

The forward rate is a stochastic variable. In the K-factor HJM-model [11, 12, 13] the time evolution for the forward rates is given by (sum over all repeated index)

$$
\frac{\partial f}{\partial t}(t, x)=\alpha(t, x)+\sigma_{i}(t, x) W_{i}(t)
$$

where $\alpha(t, x)$ is the drift velocity term and $\sigma_{i}(t, x)$ is the deterministic volatility for the forward rates. From eqn.(2.4) we have

$$
f(t, x)=f\left(t_{0}, x\right)+\int_{t_{0}}^{t} d t^{\prime} \alpha\left(t^{\prime}, x\right)+\int_{t_{0}}^{t} d t^{\prime} \sigma_{i}\left(t^{\prime}, x\right) W_{i}\left(t^{\prime}\right)
$$

The initial forward rate $f\left(t_{0}, x\right)$ is determined from the market, and so are the volatility functions $\sigma_{i}(t, x)$.

Each stochastic variable $W_{i}(t), i=1,2 \ldots K$ is an independent Gaussian white noise given by

$$
E\left(W_{i}(t) W_{j}\left(t^{\prime}\right)\right)=\delta_{i j} \delta\left(t-t^{\prime}\right)
$$

Note that the forward rates $f(t, x)$ are driven by random variables $W_{i}(t)$ which gives the same random 'shock to all the forward rates; the volatility function $\sigma(t, x)$ weighs this 'shock' differently for each time $t$ and each $\mathrm{x}$. It is precisely this feature which we will generalize later such that $f(t, x)$ is taken to be an independent random variable for each $x$ and each $t$.

To write the probability measure for $W_{i}(t)$ we discretize $t=m \epsilon$, with $m=1,2 \ldots M=\left[\frac{t}{\epsilon}\right]$, and where $t$ takes values in a finite interval depending on the problem of interest; then the probability measure is given by

$$
\mathcal{P}[W]=\prod_{m=1}^{M} \prod_{i=1}^{K} e^{-\frac{\epsilon}{2} W_{i}^{2}(m)}
$$




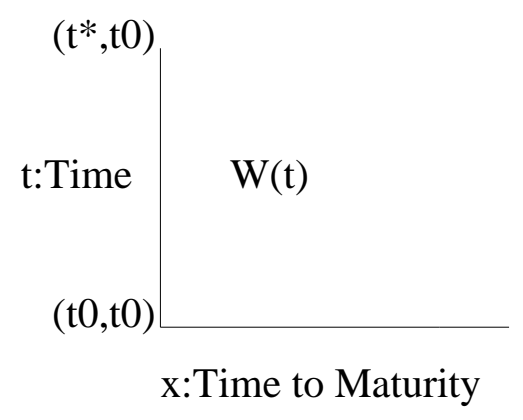

Figure 1: Domain of $\mathrm{W}(\mathrm{t})$

$$
\int d W=\prod_{m=1}^{M} \prod_{i=1}^{K} \sqrt{\frac{\epsilon}{2 \pi}} \int_{-\infty}^{+\infty} d W_{i}(m)
$$

For notational simplicity we take the limit of $\epsilon \rightarrow 0$; note that for purposes of rigor, the continuum notation is simply a short-hand for taking the continuum limit of the discrete multiple integrals given above. We have, for $t_{1}<t<t_{2}$

$$
\begin{aligned}
\mathcal{P}\left[W, t_{1}, t_{2}\right] & \rightarrow e^{S_{0}} \\
S_{0} \equiv S_{0}\left[W, t_{1}, t_{2}\right] & =-\frac{1}{2} \sum_{i=1}^{K} \int_{t_{1}}^{t_{2}} d t W_{i}(t) W_{i}(t) \\
\int d W & \rightarrow \int D W
\end{aligned}
$$

The 'action' functional $S_{0}$ is ultra-local with all the variables being decoupled; generically, $\int D W$ stands for the (path) integration over all the random variables $W(t)$ which appear in the problem. The integration variables $W(t)$ are shown in Fig.(而).

A path integral approach to the HJM-model has been discussed in [14]; the action they derive is different than the one given above since they use a different set of variables and end up with an action involving the time derivatives of their variables.

A formula that we will repeatedly need is the generating functional for $W$ given by the path integral 


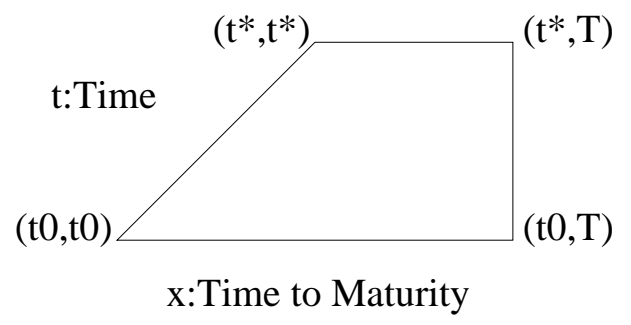

Figure 2: Trapezoidal Domain $\mathcal{T}$

$$
\begin{aligned}
Z\left[j, t_{1}, t_{2}\right] & =\int D W e^{\int_{t_{1}}^{t_{2}} d t j_{i}(t) W_{i}(t)} e^{S_{0}\left[W, t_{1}, t_{2}\right]} \\
& =e^{\frac{1}{2} \int_{t_{1}}^{t_{2}} d t j_{i}(t) j_{i}(t)}
\end{aligned}
$$

We now derive the no arbitrage condition on the drift velocity $\alpha(t, x)$. Recall having a martingale measure is equivalent to having no arbitrage for the price of the coupon and zero-coupon bonds [15]. The martingale condition states the following: suppose a zero-coupon bond which matures at time $T$ has a price of $P\left(t_{*}, T\right)$ at time $t_{*}$ and at time $t_{0}<t_{*}$ has a price $P\left(t_{0}, T\right)$; then the price of the bond at $t_{*}$, evolved backward to time $t_{0}$ and continuously discounted by the risk-free spot rate $r(t)$ must be equal to the price of the bond at time $t_{0}$.

In other words, the martingale condition on the zero-coupon bond using (2.5) is given by

$$
\begin{aligned}
P\left(t_{0}, T\right) & =E_{t_{0}}\left[e^{-\int_{t_{0}}^{t_{*}} r(t) d t} P\left(t_{*}, T\right)\right] \\
& =P\left(t_{0}, T\right) e^{-\int_{\mathcal{T}} \alpha(t, x)} \int D W e^{-\int_{\mathcal{T}} \sigma_{i}(t, x) W(t)} e^{S_{0}[W]}
\end{aligned}
$$

where the trapezoidal domain $\mathcal{T}$ is given in Fig.(2) and

$$
\int_{\mathcal{T}} \equiv \int_{t_{0}}^{t_{*}} d t \int_{t}^{T} d x
$$

We can set $t_{*}=T$ in above equation; this will change the domain to a (right isosceles) triangular domain $\Delta$ given in Fig.(3) and is the largest domain in the problem. We have, using $P(T, T)=1$, the following 


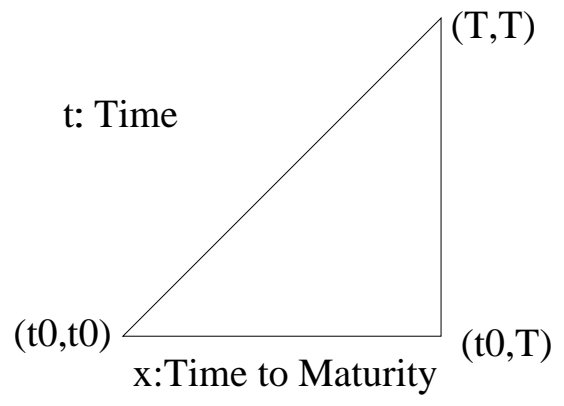

Figure 3: Maximum Domain $\Delta$

$$
\begin{aligned}
P\left(t_{0}, T\right) & =E_{t_{0}}\left[e^{-\int_{t_{0}}^{t_{*}} r(t) d t}\right] \\
\Rightarrow e^{\int_{\Delta} \alpha(t, x)} & =\int D W e^{-\int_{\Delta} \sigma_{i}(t, x) W(t)} e^{S_{0}[W]}
\end{aligned}
$$

On performing the $\mathrm{W}$-integrations we obtain from (2.14)

$$
e^{-\int_{\mathcal{T}^{\alpha}} \alpha(t, x)}=e^{\frac{1}{2} \int_{t_{0}}^{t *} d t\left[\int_{t}^{T} d x \sigma(t, x)\right]^{2}}
$$

Dropping the integration over $t$ we obtain [12]

$$
\int_{t}^{T} d x \alpha(t, x)=\frac{1}{2} \sum_{i=1}^{K}\left[\int_{t}^{T} d x \sigma_{i}(t, x)\right]^{2}
$$

or equivalently

$$
\begin{aligned}
\alpha(t, x) & =\sigma_{i}(t, x) \int_{t}^{x} d y \sigma_{i}(t, y) \\
& : \text { Conditionfor NoArbitrage }
\end{aligned}
$$

We have see that, as expected, the martingale condition leads to the well-known no arbitrage condition on the drift velocity of the forward rates.

Consider the 2-Factor HJM-model with volatilities given by

$$
\sigma_{1}(t, x)=\sigma_{1} ; \sigma_{2}(t, x)=\sigma_{2} e^{-\lambda(x-t)}
$$


The no arbitrage condition given in eqn.(2.20) for this case yields

$$
\alpha(t, x)=\sigma_{1}^{2}(x-t)+\frac{\sigma_{2}^{2}}{\lambda} e^{-\lambda(x-t)}\left(1-e^{-\lambda(x-t)}\right)
$$

\section{$3 \quad$ Futures Pricing in the HJM-Model}

The future and forward contracts on a zero-coupon coupon bond are instruments that are traded in the capital markets [12, [13]. The forward and future price of $P(t, T)$, namely $F\left(t_{0}, t_{*}, T\right)$ and $\mathcal{F}\left(t_{0}, t_{*}, T\right)$ respectively, is the price fixed at time $t_{0}<t_{*}$ for having a zero-coupon bond delivered to the buyer at time $t_{*}$.

The difference in the two instruments is that for a forward contract there is only a single cash flow at the expiry date of the contract $t_{*}$. For a futures contract on the other hand there is a continuous cash flow from time $t_{0}$ to $t_{*}$ such that all variations in the price of $P(t+d t, T)$ away from $P(t, T)$, for $t_{0}<t<t_{*}$, is settled continuously between the buyer and the seller, with a final payment of $P\left(t_{*}, T\right)$ at time $t_{*}$ [12, 13]. If the time-evolution of $P(t, T)$ was deterministic, it is easy to see that the forward and futures price would be equal.

It can be shown that the price of the futures $\mathcal{F}$ is given by 12

$$
\mathcal{F}\left(t_{0}, t_{*}, T\right)=E_{t_{0}}\left[P\left(t_{*}, T\right)\right]
$$

From eqns.(2.5) and (2.9) we have

$$
\begin{aligned}
\mathcal{F}\left(t_{0}, t_{*}, T\right) & =\int D W e^{-\int_{t_{*}}^{T} d x f\left(t_{*}, x\right)} \mathcal{P}\left[W, t_{0}, t_{*}\right] \\
& =F\left(t_{0}, t_{*}, T\right) \exp \Omega_{\mathcal{F}}
\end{aligned}
$$

where the forward price for the same contract is given by

$$
F\left(t_{0}, t_{*}, T\right)=\frac{P\left(t_{0}, T\right)}{P\left(t_{0}, t_{*}\right)}
$$

The trapezoidal domain $\mathcal{T}$ splits into a triangle and a rectangle shown in Fig.(田) and yields

$$
\mathcal{T}=\Delta_{0} \oplus \mathcal{R}
$$




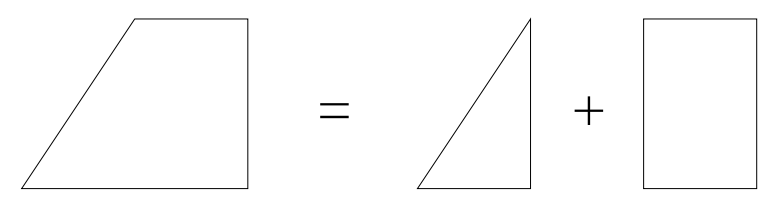

Figure 4: Domain $\mathcal{T}=$ Domain $\Delta_{0} \oplus$ Domain $\mathcal{R}$

The futures price is defined over the rectangular domain $\mathcal{R}$ given in Fig.(牙) and

$$
\int_{\mathcal{R}} \equiv \int_{t_{0}}^{t_{*}} d t \int_{t_{*}}^{T} d x
$$

We have

$$
\exp \Omega_{\mathcal{F}}=e^{\Omega} e^{-\int_{\mathcal{R}} \alpha(t, x)}
$$

with

$$
\begin{aligned}
e^{\Omega} & =\int D W e^{-\int_{\mathcal{R}} \sigma_{i}(t, x) W_{i}(t)} e^{S_{0}} \\
& =\exp \left\{\frac{1}{2} \sum_{i=1}^{K} \int_{t_{0}}^{t_{*}} d t\left[\int_{t_{*}}^{T} d x \sigma_{i}(t, x)\right]^{2}\right\}
\end{aligned}
$$

where eqn. (3.9) has been obtained by performing the path integration over the $W$-variables using eqn. (2.12).

Collecting results from above we have, using the no arbitrage condition from eqn. (2.20) and after some simplifications

$$
\Omega_{\mathcal{F}}\left(t_{0}, t_{*}, T\right)=-\int_{t_{0}}^{t_{*}} d t \int_{t}^{t_{*}} d x \sigma_{i}(t, x) \int_{t_{*}}^{T} d x^{\prime} \sigma_{i}\left(t, x^{\prime}\right)
$$

As is expected, the future and forward prices of the zero-coupon bond are equal if the volatility is zero, that is, the evolution of the zero-coupon bond is deterministic.

Consider the 2-Factor HJM-model with volatilities given in eqn. (2.21). Equation (3.10) yields 


$$
\begin{aligned}
\Omega_{\mathcal{F}}\left(t_{0}, t_{*}, T\right) & =-\sigma_{1}^{2}\left(T-t_{*}\right)\left(t_{*}-t_{0}\right)^{2} \\
& -\frac{\sigma_{0}^{2}}{2 \lambda^{3}}\left(1-e^{-\lambda\left(T-t_{*}\right)}\right)\left(1-e^{-\lambda\left(t_{*}-t_{0}\right)}\right)^{2}
\end{aligned}
$$

which is the result given in [1].

\section{Option and Cap Pricing in the HJM-Model}

Suppose we need the price at time $t_{0}$ of a derivative instrument of a zerocoupon bond $P(t, T)$ for a contract which expires at $t_{*}$ 12, 13. For concreteness we study the price of a European call option on a zero-coupon bond, namely $C\left(t_{0}, t_{*}, T, K\right)$; the option has a strike price of $K$ and exercise time at $t_{*}>t_{0}$.

The final value of the option at $t_{0}=t_{*}$ is, as required by the contract, given by

$$
\begin{aligned}
C\left(t_{*}, t_{*}, T, K\right) & =\left(P\left(t_{*}, T\right)-K\right)_{+} \\
& \equiv\left(P\left(t_{*}, T\right)-K\right) \theta\left(P\left(t_{*}, T\right)-K\right)
\end{aligned}
$$

where the step function is defined by

$$
\theta(x)= \begin{cases}1 & \text { for } \mathrm{x}>0 \\ \frac{1}{2} & \text { for } \mathrm{x}=0 \\ 0 & \text { for } \mathrm{x}<0\end{cases}
$$

For $t_{0}<t_{*}$ we have the price of $C$ given by

$$
C\left(t_{0}, t_{*}, T, K\right)=E_{t_{0}}\left[e^{-\int_{t_{0}}^{t_{*}} d t f(t, t)}\left(P\left(t_{*}, T\right)-K\right)_{+}\right]
$$

The expectation value in eqn.(4.4) is taken by evolving the payoff function $\left(P\left(t_{*}, T\right)-K\right)_{+}$backward to $t_{0}$, discounted by stochastic spot rate $r(t)=$ $f(t, t)$.

Using the identity

$$
\delta(z)=\frac{1}{2 \pi} \int_{-\infty}^{+\infty} d p e^{i p z}
$$


we can rewrite eqn. (4.4) as

$$
C\left(t_{0}, t_{*}, T, K\right)=\int_{-\infty}^{+\infty} d G \Psi\left(G, t_{*}, T\right)\left(e^{G}-K\right)_{+}
$$

where

$$
\begin{aligned}
\Psi\left(G, t_{*}, T\right) & =E_{t_{0}}\left[e^{-\int_{t_{0}}^{t_{*}} d t f(t, t)} \delta\left(P\left(t_{*}, T\right)-e^{G}\right)\right] \\
& =\int_{-\infty}^{+\infty} \frac{d p}{2 \pi} e^{\Lambda} e^{i p\left(G+\Lambda_{0}\right)} \\
\Lambda_{0} & =\ln F\left(t_{0}, t_{*}, T\right)
\end{aligned}
$$

Using (2.2) and (2.5), we have the following

$$
e^{\Lambda}=\frac{\int D W e^{-\int_{\Delta_{0}} \sigma_{i}(t, x) W_{i}(t)+i p \int_{\mathcal{R}} \sigma_{i}(t, x) W_{i}(t)} e^{S_{0}}}{e^{-\int_{\Delta_{0}} \alpha(t, x)+i p \int_{\mathcal{R}} \alpha(t, x)}}
$$

Note the interplay of the subdomains $\Delta_{0}$ and $\mathcal{R}$ in determining the price of the option. Using (2.12) to perform the integrations over $W$ yields, after considerable simplifications and using the no arbitrage condition given by eqn. (2.19) the following

$$
\Lambda=-\frac{q^{2}}{2}\left(p^{2}+i p\right)
$$

with

$$
q^{2}=\sum_{i=1}^{K} \int_{t_{0}}^{t_{*}} d t\left[\int_{t_{*}}^{T} d x \sigma_{i}(t, x)\right]^{2}
$$

To obtain eqn. (4.11) we have used the identity

$$
\int_{t_{0}}^{t_{*}} d t\left[\int_{t_{*}}^{T} d x \alpha(t, x)-\int_{t}^{t_{*}} d x \sigma_{i}(t, x) \int_{t_{*}}^{T} d y \sigma_{i}(t, y)\right]=\frac{1}{2} q^{2}
$$

Performing the Gaussian integration in eqn.(4.8) yields 


$$
\Psi\left(G, t_{*}, T\right)=\sqrt{\frac{1}{2 \pi q^{2}}} \exp -\frac{1}{2 q^{2}}\left\{G+\int_{t_{*}}^{T} d x f\left(t_{0}, x\right)-\frac{q^{2}}{2}\right\}^{2}
$$

Hence from above and (4.6) we recover the well-known result 17, 18 that the European option on a zero-coupon has a Black-Scholes like formula with volatility given by $q$.

For the 2-Factor HJM-model given in eqn. 22.21) we have

$$
\begin{aligned}
q^{2} & =\sigma_{1}^{2}\left(T-t_{*}\right)^{2}\left(t_{*}-t_{0}\right) \\
& +\frac{\sigma_{2}^{2}}{2 \lambda^{3}}\left(1-e^{-\lambda\left(T-t_{*}\right)}\right)^{2}\left(1-e^{-2 \lambda\left(t_{*}-t_{0}\right)}\right)
\end{aligned}
$$

A cap is a financial instrument for reducing ones exposure to interest rate fluctuations, and guarantees a maximum interest rate for borrowings over a fixed time. A cap fixes the maximum interest for a fixed period from $t_{*}$ to $t_{*}+T$; the buyer of the instrument then pays for this period the maximum of the Libor (London interbank offered rate) $L \equiv L\left(t_{*}, t_{*}+T\right)$ or the cap rate $K$. The cap is exercised at time $t_{*}$ and the payments are made, in arrears, at time $t_{*}+T$. Let the principal amount be $V$; the value of the cap at time $t_{*}$ is then given by

$$
\operatorname{Cap}\left(t_{*}, t_{*}, T\right)=V T(L-K)_{+}
$$

We have in terms of the forward rates [8]

$$
\begin{aligned}
T L\left(t_{*}, t_{*}+T\right) & =e^{\int_{t_{*}}^{T+t_{*}} d x f\left(t_{*}, x\right)}-1 \\
& =\frac{1}{P\left(t_{*}, t_{*}+T\right)}-1
\end{aligned}
$$

The price of the cap at time $t_{0}<t_{*}$ is given by

$$
\begin{aligned}
C a p\left(t_{0}, t_{*}, T, X\right) & =V E_{t_{0}}\left[e^{-\int_{t_{0}}^{t_{*}} d t f(t, t)}\left(\frac{1}{P\left(t_{*}, t_{*}+T\right)}-1-T K\right)_{+}\right] \\
& =V \int_{-\infty}^{+\infty} d H \Gamma(H)\left(e^{H}-1-T K\right)_{+}
\end{aligned}
$$


Carrying out an analysis similar to the one done for the pricing of the European call option we obtain, as in (4.14) (note the minus sign of $\mathrm{H}$ )

$$
\begin{aligned}
\Gamma(H) & =\Psi\left(-H, t_{*}, T+t_{*}\right) \\
& =\sqrt{\frac{1}{2 \pi q_{c a p}^{2}}} \exp -\frac{1}{2 q_{c a p}^{2}}\left(-H+\int_{t_{*}}^{T+t_{*}} d x f\left(t_{0}, x\right)-\frac{q_{c a p}^{2}}{2}\right)^{2}
\end{aligned}
$$

with $q_{\text {cap }}$ for the Two-Factor model given similar to (4.15) by

$$
\begin{aligned}
q_{\text {cap }}^{2} & =\sigma_{1}^{2} T^{2}\left(t_{*}-t_{0}\right) \\
& +\frac{\sigma_{2}^{2}}{2 \lambda^{3}}\left(1-e^{-\lambda T}\right)^{2}\left(1-e^{-2 \lambda\left(t_{*}-t_{0}\right)}\right)
\end{aligned}
$$

The formula above shows that a cap is equivalent to a European put option on the zero-coupon bond $P\left(t_{*}, t_{*}+T\right)$. For a caplet the time $T$ is taken to be small so that $L\left(t_{*}, t_{*}+T\right) \simeq f\left(t_{*}, t_{*}\right)$, and in eqn. (4.22) we have

$$
\begin{aligned}
\int_{t_{*}}^{T+t_{*}} d x f\left(t_{0}, x\right) & \simeq T f\left(t_{0}, t_{*}\right) \\
q_{\text {cap }}^{2} & \simeq T^{2}\left[\sigma_{1}^{2}\left(t_{*}-t_{0}\right)+\frac{\sigma_{2}^{2}}{2 \lambda}\left(1-e^{-2 \lambda\left(t_{*}-t_{0}\right)}\right)\right]
\end{aligned}
$$

\section{Quantum Field Theory of Treasury Bonds}

As mentioned earlier, in the HJM-model the fluctuations in the forward rates at a given time $t$ are given by 'shocks' which are delivered to the whole curve $f(t, x)$ by a single random variable $W(t)$; clearly, a more general evolution of the instantaneous forward rate would be to let the whole curve evolve randomly, that is let all the forward rates fluctuate independently. The only constraint on the random evolution is that for every instant there be no arbitrage for the forward rates.

Since there are infinitely many forward rates, we need an infinite number of independent variables to describe the random evolution of the yield curve. As mentioned earlier the generic quantity describing such a system 
is a quantum field [6]; for modeling Treasury Bonds we will need to study a two-dimensional quantum field in a finite Euclidean domain.

We consider the forward rates to be a quantum field; $f(t, x)$ is taken to be an independent random variable for each $x$ and each $t$. For notational simplicity we keep both $t$ and $x$ continuous; in Appendix A, the case with both $t$ and $x$ discrete is analyzed and the continuum limit discussed in some detail.

For the sake of concreteness, consider the price of an options contract (at time $t_{0}$ ) of a $T$-maturity zero-coupon bond ; let the contract expire at time $t_{*}$. Since the all the forward rates are for the future, we always have $x>t$; hence the quantum field $f(t, x)$ is defined on the trapezoidal domain $\mathcal{T}$ bounded by $t=t_{0}, t=x, t=t_{*}$ and $x=T$, as shown in Fig (2).

We introduce a new parameter $\mu$ which quantifies the strength of the fluctuations in the time-to-maturity direction $x$; we expect that in the limit of $\mu \rightarrow 0$, we should recover the HJM-model. The simplest term which can control the fluctuations in the $x$-direction is the gradient of $f(t, x)$ with respect to $x$. The action given in eqn.(2.10) is generalized to

$$
\begin{aligned}
& S[f]=\int_{\mathcal{T}} \mathcal{L}[f] \\
& \mathcal{L}[f]=-\frac{1}{2(T-t)}\left[\left\{\frac{\frac{\partial f(t, x)}{\partial t}-\alpha(t, x)}{\sigma(t, x)}\right\}^{2}+\frac{1}{\mu^{2}}\left\{\frac{\partial}{\partial x}\left(\frac{\frac{\partial f(t, x)}{\partial t}-\alpha(t, x)}{\sigma(t, x)}\right)\right\}^{2}\right]
\end{aligned}
$$

The initial condition is given by

$$
t=t_{0}, t_{0}<x<T: f\left(t_{0}, x\right): \text { specified }
$$

and the field values of $f(t, x)$ on the rest of the boundary points of the trapezoid $\mathcal{T}$ are arbitrary and are integration variables. The quantum field theory is defined by integrating over all configurations of $f(t, x)$ and yields

$$
\begin{aligned}
Z & =\int D f e^{S[f]} \\
\int D f & =\prod_{(t, x) \in \mathcal{T}} \int_{-\infty}^{+\infty} d f(t, x)
\end{aligned}
$$


Note that $e^{S[f]} / \mathrm{Z}$ is the probability for different field configurations to occur when the functional integral over $f(t, x)$ is performed.

The presence of the second term in the action given in (5.1) seems to be justified from the phenomenology of the forward rates [19 and is not ruled out by no arbitrage. This term in the action also implies that all the random configurations of $f(t, x)$ which appear in the path integral (5.4) are continuous functions of $x$. Forward rates that are usually observed in the market are continuous $[7]$.

However the random configurations for the forward rates are nowhere differentiable. It is noted in [7] that there is no evidence to indicate whether the actual forward rates are differentiable or not.

The action given above is suitable for studying formal properties of the forward rates. However it is often simpler for computational purposes to change variables. Let $A(t, x)$ be a two-dimensional quantum field; we use the HJM-change of variables to express $A(t, x)$ in terms of the forward rates $f(t, x)$, namely

$$
\frac{\partial f}{\partial t}(t, x)=\alpha(t, x)+\sigma(t, x) A(t, x)
$$

The Jacobian of the above transformation is a constant and hence can be ignored. The action can now be written as

$$
\begin{aligned}
S[A] & =-\frac{1}{2} \int_{t_{0}}^{t_{*}} d t \frac{1}{T-t} \int_{t}^{T} d x\left\{A^{2}(t, x)+\frac{1}{\mu^{2}}\left(\frac{\partial A(t, x)}{\partial x}\right)^{2}\right\} \\
& =\int_{\mathcal{T}} \mathcal{L}[A]
\end{aligned}
$$

The quantum field theory is defined by a functional integral over all variables $A(t, x)$; the values of $A(t, x)$ on the boundary of $\mathcal{T}$ are arbitrary and are integration variables; this yields the partition function

$$
Z=\int D A e^{S[A]}
$$

Note that eqns. (5.6) and (5.7) can easily be generalized to the K-Factor case. It is shown in eqn. A.15) that if we define

$$
W(t)=\frac{1}{T-t} \int_{t}^{T} d x A(t, x)
$$


then for $\mu \rightarrow 0$ we have

$$
\begin{aligned}
S[A] & \rightarrow S_{0}=-\frac{1}{2} \int_{t_{0}}^{t_{*}} d t W^{2}(t) \\
\int D A & \rightarrow \int D W
\end{aligned}
$$

From eqn.(2.10) and above we see that we recover the HJM-model in the $\mu \rightarrow 0$ limit. We see from eqn.(5.10) that the HJM-model is a drastic truncation of the full field theory and only considers the fluctuations of the average value of the quantum field $A(t, x)$; it in effect 'freezes-out' all the other fluctuations of $A(t, x)$.

If one thinks of the field $A\left(t_{0}, x\right)$ at some instant $t_{0}$ as giving the position of a 'string' [7, 8], then in the HJM-model this string is taken to be a rigid string. The action $S[A]$ given in (5.7) allows all the degrees of freedom of the field $A\left(t_{0}, x\right)$ to fluctuate independently and can be thought of as a 'string' with string tension equal to $\frac{1}{\mu^{2}}$; in this language the HJM-model considers the interest yield curve to be a string with infinite tension and hence rigid.

The moment generating functional for the quantum field theory is given by the Feynman path integral as

$$
Z[J]=\frac{1}{Z} \int D A e^{\int_{t_{0}}^{t_{*}} d t \int_{t}^{T} d x J(t, x) A(t, x)} e^{S[A]}
$$

We evaluate $Z[J]$ exactly in Appendix B, and from eqn.(B.17)

$$
Z[J]=\exp \frac{1}{2} \int_{t_{0}}^{t_{*}} d t \int_{t}^{T} d x d x^{\prime} J(t, x) D\left(x, x^{\prime} ; t, T\right) J\left(t, x^{\prime}\right)
$$

where the propagator $D\left(x, x^{\prime} ; t, T\right)$ is given from eqn.(B.19), for $\beta=T-t$, by

$$
\begin{aligned}
D\left(x, x^{\prime} ; t, T\right) & =\frac{\mu \beta}{\sinh ^{3}(\mu \beta)}\left[\sinh \mu(T-x) \sinh \mu\left(x^{\prime}-t\right)\left\{1+\sinh ^{2}(\mu \beta) \theta\left(x-x^{\prime}\right)\right\}\right. \\
& +\sinh \mu\left(T-x^{\prime}\right) \sinh \mu(x-t)\left\{1+\sinh ^{2}(\mu \beta) \theta\left(x^{\prime}-x\right)\right\} \\
& +\cosh (\mu \beta)\left\{\sinh \mu(x-t) \sinh \mu\left(x^{\prime}-t\right)\right. \\
& \left.\left.+\sinh \mu(T-x) \sinh \mu\left(T-x^{\prime}\right)\right\}\right]
\end{aligned}
$$


To understand the significance of the propagator $D\left(x, x^{\prime} ; t, T\right)$ note that the correlator of the field $A(t, x)$, for $t_{0}<t, t^{\prime}<t_{*}<T$, is given by

$$
\begin{aligned}
E\left(A(t, x) A\left(t^{\prime}, x^{\prime}\right)\right) & =\frac{1}{Z} \int D A e^{S[A]} A(t, x) A\left(t^{\prime}, x^{\prime}\right) \\
& =\delta\left(t-t^{\prime}\right) D\left(x, x^{\prime} ; t, T\right)
\end{aligned}
$$

In other words, $D\left(x, x^{\prime} ; t, T\right)$ is a measure of the effect of a value of field $A(t, x)$ at maturity $x$ on its value at another maturity $x^{\prime}$.

Since $D\left(x, x^{\prime} ; t, T\right)$ looks fairly complicated, we examine it in a few extreme limits. In the limit of $\mu \rightarrow 0$ we have

$$
D\left(x, x^{\prime} ; t, T\right)=1+O\left(\mu^{2}\right)
$$

We see that, as expected, all the fluctuations in the $x$ direction are exactly correlated; in other words the values of $A(t, x)$ for different maturities are all the same. Defining

$$
j(t)=\int_{t}^{T} d x J(t, x)
$$

we have from eqns. (5.14) and (5.18) that

$$
Z[j]=\exp \frac{1}{2} \int_{t_{0}}^{t_{*}} d t j^{2}(t)
$$

which is the result obtained earlier in eqn.(2.12).

For $\mu \rightarrow \infty$ we have

$$
D\left(x, x^{\prime} ; t, T\right) \simeq \frac{1}{2} \mu \beta e^{-\mu\left|x-x^{\prime}\right|}
$$

The propagator above has a simple interpretation; if the field $A(t, x)$ has some value at point $x$, then the field at 'distances' $x-\mu^{-1}<x^{\prime}<x+\mu^{-1}$ will tend to have the same value, whereas for other values of $x^{\prime}$ the field will have arbitrary values. Hence we see in this limit that the fluctuations in the time-to-maturity $x$ direction are strongly correlated within maturity time $\mu^{-1}$, which is the correlation time of the forward rates.

We now derive the no-arbitrage condition for the action $S[A]$. Eqn.(2.13) for the martingale is unchanged; generalizing eqns. (2.14) and (2.18) we have 


$$
\begin{aligned}
\exp \int_{\mathcal{T}} \alpha(t, x) & =\frac{1}{Z} \int D A e^{-\int_{\mathcal{T}} \sigma(t, x) A(t, x)} e^{\int_{\mathcal{T}} \mathcal{L}[A]} \\
& =\exp \frac{1}{2} \int_{t_{0}}^{t_{*}} d t \int_{t}^{T} d x d x^{\prime} \sigma(t, x) D\left(x, x^{\prime} ; t, T\right) \sigma\left(t, x^{\prime}\right)
\end{aligned}
$$

Hence we have

$$
\int_{t}^{T} d x \alpha(t, x)=\frac{1}{2} \int_{t}^{T} d x d x^{\prime} \sigma(t, x) D\left(x, x^{\prime} ; t, T\right) \sigma\left(t, x^{\prime}\right)
$$

which is the generalization of eqn.(2.19), and that of eqn. (2.20) is given by

$$
\begin{aligned}
\alpha(t, T) & =\sigma(t, T) \int_{t}^{T} d x^{\prime} D\left(T, x^{\prime} ; t, T\right) \sigma\left(t, x^{\prime}\right) \\
& +\frac{1}{2} \int_{t}^{T} d x d x^{\prime} \sigma(t, x) \frac{\partial D\left(x, x^{\prime} ; t, T\right)}{\partial T} \sigma\left(t, x^{\prime}\right)
\end{aligned}
$$

From the empirical study of forward rate curves, there is evidence 19 that the naive HJM-model no arbitrage for the drift term $\alpha(t, x)$ is not adequate since it is quadratic in the volatility; in [19] an additional term is added which reflects the market price of risk. In the approach of field theory, the additional term involving the derivative of the propagator could provide a better model of no arbitrage for the drift term.

For $\mu \rightarrow \infty$ we have

$$
\alpha(t, T)=\frac{1}{2}(T-t) \sigma^{2}(t, T)+\frac{1}{2} \int_{t}^{T} d x \sigma^{2}(t, x)
$$

Note the expression for $\alpha(t, x)$ given above is quite dissimilar from that of the HJM-model given in eqn. (2.20), which is the case for $\mu=0$; the values of $\alpha(t, x)$ given in (5.25) for $\mu \neq 0$ continuously interpolate between the extreme values of $\mu=0$ and $\mu=\infty$.

For the Two-Factor case given in eqn.(2.21), we can exactly solve for $\alpha(t, x)$ in terms of the volatilities; the expressions are long and cumbersome. For the case of the One-Factor model with only $\sigma_{1} \neq 0$, we have the exact result that 


$$
\alpha(t, x)=\sigma_{1}^{2}(x-t)
$$

which is independent of $\mu$ and the same as the HJM-model. This result can be seen directly from the functional integral; since $\sigma_{1}=$ constant, in the no arbitrage eqn. (5.22) for $\alpha(t, x)$, we see that it only couples to $\int_{t}^{T} d x A(t, x)=$ $(T-t) W(t)$; a change of variables then shows that $\alpha(t, x)$ does not couple to $\mu$, and hence the simple result. As we will see later, the One-Factor model has non-trivial dependence on $\mu$ for other quantities such as futures and options.

We have from eqns.(5.6), (5.25)

$$
\begin{aligned}
f(t, x) & =f\left(t_{0}, x\right)+\frac{1}{2} \int_{t_{0}}^{t} d t^{\prime} \int_{t^{\prime}}^{x} d y d y^{\prime} \sigma\left(t^{\prime}, y\right) \frac{\partial D\left(y, y^{\prime} ; t^{\prime}, x\right)}{\partial x} \sigma\left(t^{\prime}, y^{\prime}\right) \\
& +\int_{t_{0}}^{t} d t^{\prime} \sigma\left(t^{\prime}, x\right)\left[\int_{t^{\prime}}^{x} d y^{\prime} D\left(x, y^{\prime}\right) \sigma_{i}\left(t^{\prime}, y^{\prime}\right)+A\left(t^{\prime}, x\right)\right]
\end{aligned}
$$

\section{$6 \quad$ Futures and Option Pricing}

We derive the futures and options pricing using quantum field theory. For the Two-Factor model all the expressions can be obtained exactly; the results for the $\mu=0$ limit are the same as the HJM-model; we will explicitly give the results only for the case of $\mu \rightarrow \infty$ because the expressions for general $\mu$ don't add much to ones understanding.

Equation (3.7) for the futures price $\mathcal{F}$ only changes for $\Omega$; from eqn. 3.8) we have (note different domains $\mathcal{R}$ and $\mathcal{T}$ below)

$$
\begin{aligned}
e^{\Omega} & =\frac{1}{Z} \int D A e^{-\int_{\mathcal{R}} d x \sigma(t, x) A(t, x)} e^{\int_{\mathcal{T}} \mathcal{L}[A]} \\
& =\exp \left\{\frac{1}{2} \int_{t_{0}}^{t_{*}} d t \int_{t_{*}}^{T} d x d x^{\prime} \sigma(t, x) D\left(x, x^{\prime} ; t, T\right) \sigma\left(t, x^{\prime}\right)\right\}
\end{aligned}
$$

and, using the no arbitrage condition (5.25) we obtain the generalization of (3.10) given by

$$
\Omega_{\mathcal{F}}\left(t_{0}, t_{*}, T\right)=-\int_{t_{0}}^{t_{*}} d t \int_{t}^{t_{*}} d x \sigma_{i}(t, x) \int_{t_{*}}^{T} d x^{\prime} D\left(x, x^{\prime} ; t, T\right) \sigma_{i}\left(t, x^{\prime}\right)
$$


For the One-Factor Model with only $\sigma_{1} \neq 0$ we have

$$
\lim _{\mu \rightarrow \infty} \Omega_{\mathcal{F}}\left(t_{0}, t_{*}, T\right)=-\frac{\sigma_{1}^{2}}{4 \mu}\left(t_{*}-T\right)\left(2 T-t_{0}-t_{*}\right)+O\left(\frac{1}{\mu^{2}}\right)
$$

For the price of a European call option $C$, a calculation similar to the one carried out in Section 4 gives the same formula for $\Psi(G)$ given in eqn.(4.14) with $q^{2}$ given in eqn.(4.12) replaced by

$$
q^{2}=\int_{t_{0}}^{t_{*}} d t \int_{t_{*}}^{T} d x d x^{\prime} \sigma_{i}(t, x) D\left(x, x^{\prime} ; t, T\right) \sigma_{i}\left(t, x^{\prime}\right)
$$

We have

$$
\lim _{\mu \rightarrow \infty} q^{2}=\int_{t_{0}}^{t_{*}} d t(T-t) \int_{t_{*}}^{T} d x \sigma_{i}^{2}(x, t)
$$

For the Two-Factor model we have

$$
\begin{aligned}
\lim _{\mu \rightarrow \infty} q^{2} & =\frac{\sigma_{1}^{2}}{2}\left(T-t_{*}\right)\left(t_{*}-t_{0}\right)\left(2 T-t_{0}-t_{*}\right) \\
& +\frac{\sigma_{2}^{2}}{8 \lambda^{3}}\left[\left\{1+2 \lambda\left(T-t_{0}\right)\right\}\left(1-e^{-2 \lambda\left(t_{*}-t_{0}\right)}\right)\right. \\
& \left.-2 \lambda\left(t_{*}-t_{0}\right)\left(1-e^{-2 \lambda\left(T-t_{*}\right)}\right)\right]
\end{aligned}
$$

Note for both the futures and option prices, the presence of $\mu$ is like adding another factor to the model. However, the dependence of the derivatives on $\mu$ is quite different from that on $\lambda$; for instance the no arbitrage condition changes significantly as $\mu$ goes from small to large whereas no such effect happens in the case of $\lambda$; the prices of the derivatives also show non-trivial dependence on $\mu$.

If we are interested in pricing any path dependent option or other derivatives, it is not sufficient to know only the propagator $D\left(x, x^{\prime} ; t, T\right)$; the full structure of the action $S[A]$ is then required.

For example the payoff function of an Asian option at time $t_{0}$ on a zerocoupon bond $P(t, T)$ with exercise time $t_{*}$ is given by

$$
g[P(*, T)]=\left(\frac{1}{t_{*}-t_{0}} \int_{t_{0}}^{t_{*}} d t P(t, T)-K\right)_{+}
$$


Another example is the price of a European call option on a coupon bond $\mathcal{B}(t, T)$ given in (2.1); the payoff function is then

$$
g[\mathcal{B}]=\left(\mathcal{B}\left(t_{*}, T\right)-K\right)_{+}
$$

The payoff function $g[A]$ in both the cases above is path dependent. Expressing all the zero-coupon bonds in terms of the quantum field $A(t, x)$, the prices of such path dependent options at time $t_{0}$ are given by

$$
C\left(t_{0}, t_{*}, T, X\right)=\frac{1}{Z} \int D A e^{-\int_{t_{0}}^{t_{*}} d t r(t)} g[A] e^{S[A]}
$$

The computation above can only be performed numerically [20]; for this the functional integral over $A(t, x)$ has to be discretized, and which is briefly discussed in Appendix A.

\section{Conclusions}

We have re-formulated the theory of Treasury bonds in terms of path integration. The HJM-model has a simple path integral with an ultra-local action. The statements about martingale conditions and the evaluation of futures and options were shown to be calculable in a straightforward manner using path integration. The motivation for re-deriving the well-known results of the HJM-model was firstly to understand the path integral formulation of the quantities of interest in finance, and secondly, to then generalize these quantities to the case of quantum field theory.

The quantum field theory of Treasury bonds is more general than the HJM-model; in particular, the correlation of fluctuations of the forward rates can be easily modeled to be finite in the field theory whereas in the HJMmodel all the fluctuations are exactly correlated. From the point of view of finance, it is unreasonable to assume that the all forward rates fluctuate identically as in the HJM-model; the multi-factors in HJM-model try and capture the finite correlation in the time-to-maturity that should exist for the forward rates.

We considered a Gaussian model for the field theory generalization of the HJM-model as this is the simplest extension, and also because the model could be solved exactly. In particular, the formulae for the futures, cap 
and option price of Treasury bonds were derived and involved nontrivial correlations in the volatility of the model.

We can generalize the model to account for stochastic volatility of the forward rates. This entails introducing another quantum field for modeling the fluctuations of volatility, and is similar to the quantum mechanical treatment of volatility for a single security [5]. Stochastic volatility makes the system highly nonlinear and is treated in some detail in [23].

The best way of modeling Treasury bonds in practice is a computational and empirical question [21, 22]; only if the field theory model can be easily calibrated and yields more efficient algorithms, will it it be taken seriously by the practitioners of finance. For the more theoretical side of finance, the methodology of field theory certainly adds to the ways of studying and understanding the stochastic processes which drive the capital markets.

\section{Acknowledgments}

I am deeply grateful to Lawrence Ma for many useful and instructive discussions; most of my interest and understanding of this subject is a result of these discussions. I would like to thank Toh Choon Peng, Sanjiv Das, George Chacko and Michael Spalinski for stimulating interactions. I also thank Cumrun Vafa and the string theory group for their kind hospitality.

\section{Appendix}

\section{A Lattice Formulation}

We do a more careful and rigorous treatment of the field theory for the Treasury bonds. We first discretize the variables into a lattice of discrete points. Let $(t, x) \rightarrow(m \epsilon, n a)$, where $\epsilon$ is an infinitesimal time step and $a$ is an infinitesimal in the $x$ direction. Consider the trapezoidal domain $\mathcal{T}$ given in Fig.(目) to be bounded by integers $m=m_{0}\left(=\frac{t_{0}}{\epsilon}\right), m=m_{*}\left(=\frac{t_{*}}{\epsilon}\right), m \epsilon=n a$ and $n=N\left(=\frac{T}{a}\right)$. The integers then take values in the lattice version of the trapezoidal domain, say $\mathcal{D}$ given by

$$
\mathcal{D}=\left\{m=m_{0}, m_{0}+1, \ldots m_{*} ; n=m, m+1, \ldots . N\right\}
$$


The forward rates and quantum field yield on discretization

$$
\begin{aligned}
& f(t, x) \rightarrow f(m \epsilon, n a) \equiv f_{m n} \\
& A(t, x) \rightarrow A(m \epsilon, n a) \equiv A_{m n}
\end{aligned}
$$

and similarly for $\alpha$ and $\sigma$.

From eqn.(5.6) we have

$$
f_{m+1 n}=f_{m n}+\epsilon \alpha_{m n}+\epsilon \sigma_{m n} A_{m n}
$$

Using finite differences to discretize derivatives, the generalization of action $S_{0}$ in eqn.(2.10) is given, for $s=\sqrt{N a-m \epsilon}$, by

$$
\begin{aligned}
& S[A]=-\frac{\epsilon}{2} \sum_{m=m_{0}+1}^{m_{*}}\left\{\frac{a}{(N+1) a-m \epsilon} \sum_{n=m}^{N} A_{m n}^{2}+\frac{1}{N a-m \epsilon} \frac{a}{\mu^{2}} \sum_{n=m}^{N-1}\left(A_{m n+1}-A_{m n}\right)^{2}\right\} \\
& \int d A=\prod_{m=m_{0}+1}^{m_{*}} \sqrt{\frac{\epsilon \mu S}{2 \pi \sinh \mu s}} \prod_{n=m}^{N} \sqrt{\frac{\epsilon}{2 \pi \mu^{2} a}} \int_{-\infty}^{\infty} d A_{m n}
\end{aligned}
$$

Note the functional integral over the field $A(t, x)$ has been reduced to a finite-dimensional multiple integral over the $A_{m n}$ variables, which in the case above consists of $\left(m_{*}-m_{0}\right)\left\{N-\left(m_{0}+m_{*}-1\right) / 2\right\}$ independent variables; hence all the techniques useful for evaluating finite dimensional integrals can be used for performing the integration over $A_{m n}$.

To achieve the correct normalization, one in fact need not keep track of all the tedious pre-factors in (A.6). Instead one simply redefines the action by

$$
\begin{aligned}
e^{S[A]} & \rightarrow e^{S[A]} / Z \\
Z & =\int d A e^{S[A]}
\end{aligned}
$$

All the pre-factors in (A.6) cancel out; and more importantly, the expression $e^{S} / Z$ is correctly normalized to be interpreted as a probability distribution, and hence can be used for Monte Carlo studies of this theory. The action 
given in (A.5) is the starting point for any simulations that are required of the model including the pricing of path dependent derivatives; there are well known numerical algorithms developed in physics for numerically studying quantum fields [20].

We explicitly solve for the case of $\mu \rightarrow 0$ to see how the HJM-model emerges. For $\mu \rightarrow 0$, the second term in the action gives a product of $\delta$ functions and we have

$$
\begin{aligned}
e^{S[A]} & =e^{S_{0}} \prod_{m=m_{0}+1}^{m_{*}} \prod_{n=m}^{N-1} \delta\left(A_{m n+1}-A_{m n}\right) \\
S_{0} & =-\frac{\epsilon}{2} \sum_{m=m_{0}+1}^{m_{*}} \frac{a}{(N+1) a-m \epsilon} \sum_{n=m}^{N} A_{m n}^{2}
\end{aligned}
$$

Consider evaluating a typical expression like $Z$ in (5.14). For each $m$, there are $\mathrm{N}-\mathrm{m}+1$ integration variables $A_{m n}$; from eqn. (A.9) we see that there are $N-m \delta$-functions, leaving only one variable, say $A_{m m}$ unrestricted. For simplicity, we take $\epsilon=a$; hence we have

$$
\begin{aligned}
Z & =\prod_{m=m_{0}+1}^{m_{*}} \sqrt{\frac{\epsilon}{2 \pi}} \int d A_{m m} e^{S_{0}} \\
S_{0} & =-\frac{\epsilon}{2} \sum_{m=m_{0}+1}^{m_{*}} A_{m m}^{2}
\end{aligned}
$$

Defining $W(m)=A_{m m}$, we see from eqns. (2.9) that we have recovered the HJM-model. We can equivalently consider

$$
W(m)=\frac{1}{N-m+1} \sum_{n=m}^{N} A_{m n}
$$

and we have

$$
\lim _{\mu \rightarrow 0} W(m) \rightarrow A_{m m}
$$

Taking the continuum limit, we see that the field theory, in the $\mu \rightarrow 0$ limit reduces to 


$$
\begin{array}{r}
S_{0} \rightarrow-\frac{1}{2} \int_{t_{0}}^{t_{*}} d t W^{2}(t) \\
W(t)=\frac{1}{T-t} \int_{t}^{T} d x A(t, x)
\end{array}
$$

For the general case of $\mu \neq 0$, from eqn. A.5), taking the continuum limit of $\epsilon \rightarrow 0, a \rightarrow 0$ we finally obtain

$$
\begin{aligned}
S[A] & =-\frac{1}{2} \int_{t_{0}}^{t_{*}} d t \frac{1}{T-t} \int_{t}^{T} d x\left\{A^{2}(t, x)+\frac{1}{\mu^{2}}\left(\frac{\partial A(t, x)}{\partial x}\right)^{2}\right\} \\
\int D A & =\prod_{(t, x) \epsilon \mathcal{T}} \int d A \equiv \lim _{\epsilon \rightarrow 0, a \rightarrow 0} \prod_{m n} \int d A_{m n} \\
Z & =\int D A e^{S[A]}
\end{aligned}
$$

\section{B Generating Functional Z[J]}

Since the generating functional $Z[J]$ has been of central importance in studying the quantum field theory, for completeness we briefly discuss its derivation; all these results are well-known in physics [6] and this derivation is intended for readers from other disciplines.

Recall

$$
\begin{aligned}
Z[J] & =\frac{1}{Z} \int D A e^{S[A, J]} \\
S[A, J] & =\int_{t_{0}}^{t_{*}} d t \int_{t}^{T} d x J(t, x) A(t, x)+S[A]
\end{aligned}
$$

Since $S[A, J]$ is quadratic functional of the field $A(t, x)$, to perform the functional integration over the field, all we need to do is to find the specific configuration of $A(t, x)$, say $a(t, x)$ which maximizes $S[A, J]$; due to our choice of normalization $Z[J]$ depends only on $a(t, x)$.

Since there is no coupling in the time direction $t$, we study the solution $a(t, x)$ separately for each $\mathrm{t}$, and on the finite line interval $t<x<T$. We first study the case for which the boundary values of the field $A(t, x)$ are fixed, 
that is consider $A(t, t)=p$ and $A(t, T)=p^{\prime}$ to be held fixed; we will later integrate over $p, p^{\prime}$ as is required for the evaluation of $Z[J]$. We henceforth suppress the time variable $t$ for notational convenience.

The 'classical' (deterministic) field configuration $a(t, x) \equiv a(x)$ is defined by

$$
\begin{aligned}
& \frac{\delta S[a, J]}{\delta A(t, x)}=0 \\
& a(x=t)=p ; a(x=T)=p^{\prime}
\end{aligned}
$$

Doing a change of variables $A(t, x)=B(t, x)+a(t, x)$ and a functional Taylors expansion we have, from eqn.(B.3)

$$
S[A+a, J]=S_{c l}[a, J]+\tilde{S}[B]
$$

where due to boundary conditions given in eqn.(B.4) $\tilde{S}[B]$ is independent of $p, p^{\prime}, J$. The functional integral over the $B(t, x)$ variables gives only an overall constant which we can ignore and hence we have

$$
Z[J]=\frac{1}{Z} \int_{-\infty}^{+\infty} d p d p^{\prime} e^{S_{c l}[a, J]}
$$

We now determine $a(x)$; from (B.3) we have

$$
\begin{array}{r}
\frac{1}{\mu^{2}} \frac{\partial^{2} a(x)}{\partial x^{2}}-a(x)+(T-t) J(x)=0 \\
a(t)=p, a(T)=p^{\prime} ; t<x<T
\end{array}
$$

Since eqn. $(\mathbb{B} .7)$ is a linear, the solution for $a(x)$ is given by a sum of the solutions of the homogeneous and inhomogeneous equations; it can be verified that, for $\beta=T-t$, we have

$$
a(x)=\frac{\beta}{\sinh (\mu \beta)}\left[a_{H}(x)+a_{I H}(x)\right]
$$


with the homogeneous solution given by

$$
a_{H}(x)=\operatorname{psinh} \mu(T-x)+p^{\prime} \sinh \mu(x-t)
$$

and the inhomogeneous solution given by

$$
\begin{aligned}
a_{I H}(x) & =\mu \int_{t}^{T} d x^{\prime}\left[\theta\left(x-x^{\prime}\right) \sinh \mu(T-x) \sinh \mu\left(x^{\prime}-t\right)\right. \\
& \left.+\theta\left(x^{\prime}-x\right) \sinh \mu\left(T-x^{\prime}\right) \sinh \mu(x-t)\right] J\left(x^{\prime}\right)
\end{aligned}
$$

The 'classical' action is given by

$$
S_{c l}[a, J]=S_{1}\left[p, p^{\prime} ; J\right]+S_{2}[J]
$$

with

$$
\begin{aligned}
S_{1}\left[p, p^{\prime} ; J\right] & =-\frac{1}{2 \mu \beta \sinh (\mu \beta)}\left\{\cosh (\mu \beta)\left(p^{2}+{p^{\prime}}^{2}\right)-2 p p^{\prime}\right\}+\frac{1}{\sinh (\mu \beta)}\left[p P+p^{\prime} Q\right] \\
P & =\int_{t}^{T} d x \sinh \mu(x-t) J(x), Q=\int_{t}^{T} d x \sinh \mu(T-x) J(x)
\end{aligned}
$$

and

$$
S_{2}[J]=\frac{\mu \beta}{\sinh (\mu \beta)} \int_{t}^{T} d x d x^{\prime} \theta\left(x-x^{\prime}\right) \sinh \mu(T-x) \sinh \mu\left(x^{\prime}-t\right) J(x) J\left(x^{\prime}\right)
$$

Performing the Gaussian integrations over $p, p^{\prime}$ and restoring the time variable $t$ yields

$$
\begin{aligned}
Z[J] & =\frac{1}{Z} e^{S_{2}[J]} \int d p d p^{\prime} e^{S_{1}\left[p, p^{\prime} ; J\right]} \\
& =\exp \frac{1}{2} \int_{t_{0}}^{t_{*}} d t \int_{t}^{T} d x d x^{\prime} J(t, x) D\left(x, x^{\prime} ; t, T\right) J\left(t, x^{\prime}\right)
\end{aligned}
$$


where, from eqns.(B.13), (B.14) and (B.16) we have

$$
\begin{aligned}
D\left(x, x^{\prime} ; t, T\right) & =\frac{\mu \beta}{\sinh ^{3}(\mu \beta)}\left[\sinh \mu(T-x) \sinh \mu\left(x^{\prime}-t\right)\left\{1+\sinh ^{2}(\mu \beta) \theta\left(x-x^{\prime}\right)\right\}\right. \\
& +\sinh \mu\left(T-x^{\prime}\right) \sinh \mu(x-t)\left\{1+\sinh ^{2}(\mu \beta) \theta\left(x^{\prime}-x\right)\right\} \\
& +\cosh (\mu \beta)\left\{\sinh \mu(x-t) \sinh \mu\left(x^{\prime}-t\right)\right. \\
& \left.\left.+\sinh \mu(T-x) \sinh \mu\left(T-x^{\prime}\right)\right\}\right]
\end{aligned}
$$




\section{References}

[1] D.Lamberton, B.Lapeyre, N.Rabeau, 'Introduction to Stochastic Calculus Applied to Finance', Chapman and Hill (1996)

[2] P.Wilmott,S.Howison and J.Dewynne 'The Mathematics of Financial Derivatives' Cambridge Univ Press (1995)

[3] A.Das 'Field Theory: A Path Integral Approach', World Scientific (1993)

[4] J.-P. Bouchaud and D.Sornette, J.Phys.I France 4 (1994) 863-881; J.Phys.I 6 (1996) 167-175

[5] B.E.Baaquie' A Path Integral Approach to Option Pricing with Stochastic Volatility: Some Exact Results', J. Phys.I France (1997) 1733-1753; http://xxx.lanl.gov/cond-mat/9708178

[6] J. Zinn-Justin 'Quantum Field Theory and Critical Phenomenon', Cambridge University Press (1992)

[7] P. Santa-Clara and D.Sornette, 'The Dynamics of the Forward Interest Rate Curve with Stochastic String Shocks', http://xxx.lanl.gov/condmat/9801321 (1997)

[8] D.Sornette, 'String formulation of the Dynamics of the Forward Interest Rate Curve', http://xxx.lanl.gov/cond-mat/9802136 (1998)

[9] D.P.Kennedy,'Characterizing Gaussian Models of the Term Structure of Interest Rates', Mathematical Finance 7 (1997) 107-118

[10] P.Goldstein, 'The Term Structure of Interest Rates as a Random Field', Preprint, Ohio State University (1997)

[11] D.Heath, R.Jarrow and A.Morton 'Bond Pricing and the Term Structure of Interest Rates: A New Methodology for Contingent Claim Valuation' Econometrica 60 (1992) 77-105

[12] R.A.Jarrow 'Modelling Fixed Income Securities and Interest Rate Options' McGraw-Hill(1995) 
[13] R.Rebonato 'Interest-Rate Option Models', Wiley (1996)

[14] C.Chiarella and N.El-Hassan 'Evaluation of Derivative Security Prices in the Heath-Jarrow-Morton Framework as Path Integrals Using Fast Fourier Transform Techniques', Journal of Financial Engineering Vol6, No2 (1996) 121-147

[15] J.M.Harrison and S.Pliska 'Martingales and Stochastic Integrals in the Theory of Continuous Trading', Stochastic Processes and their Applications Vol 11 (1981) 215-260

[16] F.Jamshidian 'Forward Induction and Construction of Yield Curve Diffusion Models', The Journal of Fixed Income Securities (1991) 63-74

[17] R.J.Brenner and R.A.Jarrow 'A Simple Formula For Options on Discount Bonds', Advances in Futures and Options Research Vol 6 (1993) $45-51$

[18] F.Jamshidian 'An Exact Bond Option Formula', Journal of Finance 44 (1989) 205-209

[19] J-P Bouchaud, N.Sagna, R.Cont, N.El-Karoui and M.Potters 'Phenomenology of the Interest Rate Curve', http://xxx.lanl.gov/condmat/9712164

[20] D.W.Heerman 'Computer Simulations in Theoretical Physics', Springer Verlag (1990)

[21] K.C.Chan, G.A.Karyoli, F.A.Longstaff and A.B.Sanders 'An Empirical Comparison of Alternative Models of the Short-Term Interest Rate', Journal of Finance v47(3) (1992) 1209-1228

[22] B.Flesker 'Testing of the Heath-Jarrow-Morton/Ho-Lee Model of Interest Rate Contingent Claims Pricing', Journal of Financial and Quantiative Analysis 38 (1993) 483-495

[23] B.E.Baaquie 'Stochastic Volatility for the Forward Rates' (1998). 